\title{
Nature of Carbon-Carbon Bonding in Evaporated and Ion-Sputtered (Diamondlike) Amorphous Carbon from $(e, 2 e)$ Spectroscopy
}

\author{
Chao Gao, Yun Yu Wang, and A. L. Ritter \\ Virginia Polytechnic Institute, Blacksburg, Virginia 24061 \\ J. R. Dennison \\ Utah State University, Logan, Utah 84322 \\ (Received 1 December 1988)
}

\begin{abstract}
The lcal carbon-carbon bonding in evaporated $(e-\mathrm{C})$ and ion-sputtered $(i-\mathrm{C})$ amorphous carbon has been inv stigated by transmission electron-energy-loss spectroscopy (EELS) and by $(e, 2 e)$ spectroscopy. The EELS data indicate that the $e-\mathrm{C}$ is graphiclike and the $i$-C is diamondlike. The $(e, 2 e)$ data demonstrate that the concentration of $s p^{2}$ bonding is $\sim 100 \%$ in both materials $\left(0.85 \leq c_{s p^{2}} \leq 1.0\right.$ for $e$-C and $0.75 \leq c_{s p^{2}} \leq 1.0$ for $i-\mathrm{C}$ ). There is significant rehybridization of the $\pi$ orbital in $i$-C.

PACS numbers: $71.25 . \mathrm{Mg}, 78.40 . \mathrm{Fy}, 79.20 . \mathrm{Kz}$
\end{abstract}

The properties of amorphous carbon $(\alpha-\mathrm{C})$ range from black, soft "graphitic" films to hard, transparent "diamondlike" films depending on the preparation method and on the concentration of hydrogen in the sample. ${ }^{1,2}$ Evaporated amorphous carbon $(e-\mathrm{C})$ tends to be graphitic while ion-sputtered amorphous carbon $(i-\mathrm{C})$ prepared with low plasma power is more diamondlike. The presence of hydrogen in the sample also produces a diamondlike film. A natural model to describe this extreme range of optical and structural variation is to assume the local carbon-carbon bonding varies from predominantly $s p^{2}$ in graphiticlike films $t o$ a high proportion of $s p^{3}$ bonds in the diamondlike films. The validity of this model has been discussed extersively, ${ }^{1,3}$ but conclusive evidence for or against it does not exist. Some authors inferred a large fraction of $s p^{3}$ bonding in ion-sputtered carbon films (hydrogenated and nonhydrogenated) from an analysis of optical and transmission electron-energy-loss spectroscopy (EELS) measurements. ${ }^{4,5}$ Their arguments hinged on an earlier measurement of the $\pi$ electron oscillator strength in crystalline graphite. ${ }^{6}$ An error in this analysis has recently been discovered ${ }^{7}$ and it now appears that the relative fraction of $s p^{2}$ and $s p^{3}$ bonds cannot be determined from the complex dielectric function of $\alpha-\mathrm{C}$.

We have investigated graphitic $e-\mathrm{C}$ and diamondlike $i$-C by EELS and $(e, 2 e)$ spectroscopy and find that the bonding is predominantly $s p^{2}$ in both materials, but the electronic structure of the $\pi$ electrons in $e-\mathrm{C}$ and $i$-C is significantly different. The $\pi$-electron spectral density in $e-\mathrm{C}$ is similar to an angular average of the $\pi$-electron spectral density in crystalline graphite. However, in diamondlike $i$-C, the spectral density of the " $\pi$ electrons" shows significant $s-p$ hybridization. We suggest that this rehybridization of the $\pi$ orbital in $i-\mathrm{C}$ arises from the loss of mirror plane symmetry when the $s p^{2}$ bonded sheets are twisted and wrinkled. The admixture of $S$ character into the $\pi$ orbital as a function of the local radius of the $s p^{2}$ bonded sheets has been discussed extensively by Haddon, Brus, and Raghavachari. ${ }^{8}$ Our model is consistent with a detailed computer simulation of $\alpha-\mathrm{C}$ by Galli, Martin, and Car. ${ }^{9}$ We present EELS and $(e, 2 e)$ data to support this model.

The single-crystal graphite sample was prepared by the "scotch tape" method from natural graphite taken from mines at Ticonderoga, New York. The evaporated films were made by Arizona Carbon foil by arc evaporation from high-purity carbon rods. The conductivity of the $e-C$ films was $\sim 0.5 / \Omega \mathrm{cm}$. The ion-sputtered films were prepared by dc magnetron sputtering using a Sloan Orbitorr source which has a base pressure in the low $10^{-7}$-Torr range (cryopumping). The argon pressure for sputtering was $36 \mathrm{mTorr}$ and the sputtering power was $200 \mathrm{~W}$. The conductivity of these films was $\leq 10^{-2} / \Omega \mathrm{cm}$. The diffraction pattern for the $e-\mathrm{C}$ and $i$-C films exhibited diffusive rings which were qualitatively similar to published measurements. ${ }^{1}$

The loss function, $\operatorname{Im}(-1 / \epsilon)$, of crystalline graphite, $e-\mathrm{C}$, and $i$-C was measured from 0 to $80 \mathrm{eV}$ by EELS. The incident beam energy was $80 \mathrm{keV}$ and the energy resolution was $\sim 0.2 \mathrm{eV}$. All the data were taken at a momentum transfer of $0.1 \AA^{-1}$. The momentum vector was in the basal plane of the graphite sample. The loss function was extended to infinite energy by assuming that it was proportional to $1 / E^{3}$ for $E>80 \mathrm{eV}$. In order to scale the data, we assumed that $\operatorname{Re}(1 / \epsilon)=0$ for graphite and $e-\mathrm{C}$. The $i$-C sample was not a conductor so the data for it were scaled from an ellipsometry measurement of $\epsilon_{1}$ and $\epsilon_{2}$ at $2 \mathrm{eV}\left(\epsilon_{1}=5.8\right.$ and $\left.\epsilon_{2}=4.2\right)$. For all three samples the complex dielectric function, $\epsilon_{1}(\omega)+i \epsilon_{2}(\omega)$, was determined by a Kramers-Kronig analysis of the loss function. The real and imaginary part of the graphite dielectric function agreed with previous measurements. ${ }^{7,10}$ The oscillator strength was calcu- 


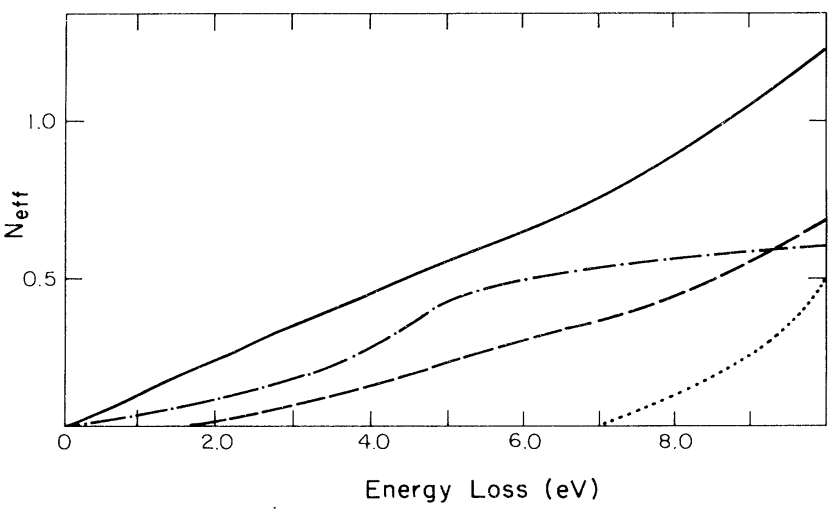

FIG. 1. The effective number of electrons (oscillator strength) as a function of energy loss for graphite (dash-dotted line), diamond (dotted line), evaporated carbon (solid line), and ion-sputtered carbon (dashed line).

lated from

$$
N_{\mathrm{eff}}(\omega)=\left(2 / \pi \omega_{p}^{2}\right) \int_{0}^{\omega} \omega^{\prime} \epsilon_{2}\left(\omega^{\prime}\right) d \omega^{\prime} .
$$

The results are shown in Fig. 1 for $\hbar \omega \leq 10 \mathrm{eV}$. The dash-dotted line is our result for graphite. The solid and dashed lines are for $e-\mathrm{C}$ and $i-\mathrm{C}$, respectively. In all three cases the oscillator strength saturates at four electrons for $\omega=\infty$. Our results for graphite are consistent with the correction of the Taft-Philipp data noted by Sonnenschein, Hanfland, and Syassen. ${ }^{7}$ The oscillator strength of diamond, the dotted line in Fig. 1, is zero below the band gap, $\sim 7 \mathrm{eV}$, then rises sharply. If the oscillator strength for the $\pi$ electrons in graphite were nearly saturated at one electron by $7 \mathrm{eV}$, as suggested by the earlier measurement, ${ }^{6}$ then the fraction of $s p^{2}$ bonding in $\alpha-\mathrm{C}$ would be simply $N_{\alpha-\mathrm{C}}(E=7 \mathrm{eV})$. The fraction of $s p^{3}$ bonds would be $1-N_{\alpha-\mathrm{C}}(E=7 \mathrm{eV})$. By this argument it was inferred that the local bonding in $e-\mathrm{C}$ is $\sim 100 \% s p^{2}$, while the bonding in an $i$-C sample, such as ours, would be $\sim 50 \% s p^{3}$. Since the $\pi$-electron oscillator strength is not saturated at the diamond band gap, the fraction of $s p^{2}$ bonds cannot be determined from $N_{\text {eff }}(7 \mathrm{eV})$. We find the same $N_{\text {eff }}(E)$ for $e-\mathrm{C}$ and $i-\mathrm{C}$ as previously measured, ${ }^{4}$ but with the corrected result for graphite it is no longer possible to separate the $\pi$ from $\sigma$ electron transitions to determine the fraction of $s p^{2}$ and $s p^{3}$ bonding. We will argue, based on the $(e, 2 e)$ data, that the fraction of $s p^{2}$ bonding is nearly the same in $e-\mathrm{C}$ and $i$-C $(\sim 100 \%)$ but that the electronic structure of the $\pi$ electrons is quite different in the two materials.

The spectral momentum density, $\rho(E, \mathbf{q})$, of crystalline and amorphous solids can be measured by $(e, 2 e)$ spectroscopy. In the independent-electron approximation the spectral density is $\rho(E, \mathbf{q})=\left|\phi_{\alpha}(\mathbf{q})\right|^{2} \delta(E$ $-E_{\alpha}$ ), where for crystalline solids $\alpha$ is a wave vector, $\mathbf{k}$, in the Brillouin zone and $\mathbf{q}=\mathbf{k}+\mathbf{G}$ ( $\mathbf{G}$ is a reciprocallattice vector). The spectral densities of crystalline

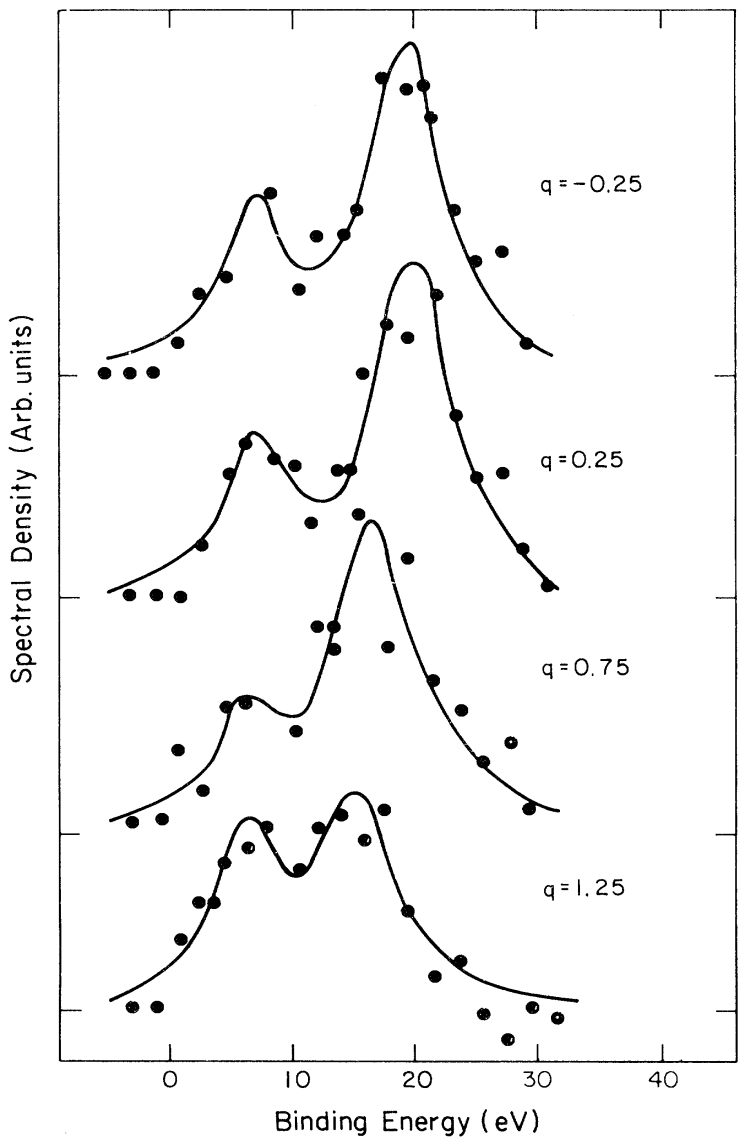

FIG. 2. The spectral density of ion-sputtered carbon as a function of binding energy (referenced to the Fermi energy) for four different momenta. The solid lines are a fit of two Lorentzians with the data.

graphite ${ }^{11}$ and evaporated carbon ${ }^{12}$ have been published. Here we report measurements on ion-sputtered films. Some of our $(e, 2 e)$ data for $i$-C are shown in Fig. 2. The spectral density as a function of energy (with respect to the Fermi energy) for four different momenta is plotted. The data are corrected for multiple scattering. ${ }^{13}$ The smaller peak at $E=8 \mathrm{eV}$ we associate with the graphite $\pi$ band and the stronger peak which disperses with momentum may be either the graphite $\sigma$ bands or a mixture of diamond and graphite $\sigma$ bands. The energy dependence of the spectra (constant momentum) has been fitted by two Lorentzian functions. The widths of the peaks are independent of $q$, within our experimental resolution, and equal to $6.60 \pm 0.5$ and $8.0 \pm 0.5 \mathrm{eV}$ for the $\pi$ and $\sigma$ peaks, respectively. The widths are primarily from incomplete deconvolution of the instrumental resolution function. We estimate the intrinsic widths of the peaks to be $\leq 2 \mathrm{eV}$. In Fig. 3 the integrated intensities of the two Lorentzian functions are represented by arrows on the energy-momentum surface. The existence of energy-momentum correlations in the 


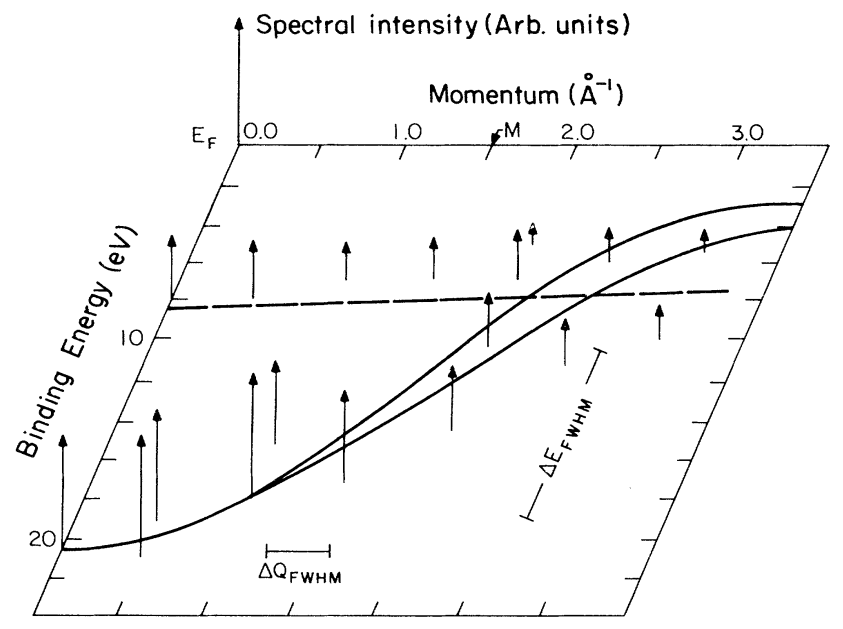

FIG. 3. The spectral intensities of ion-sputtered carbon as functions of momentum and energy (referenced to the Fermi energy). The height of the arrows is proportional to $\rho(q$, $\left.E_{\text {peak }}\right){ }^{*}$ peak width. The peak widths (FWHM) of the " $\pi$ " and " $\sigma$ " bands are 6.6 and $8 \mathrm{eV}$, respectively.

spectral momentum density of a disordered solid is a challenging conceptual problem. Some theoretical studies of simplified models for disordered solids do find that energy-momentum correlations exist in the amorphous phase. $^{14,15}$ Haydock $^{15}$ finds these correlations are similar to an angular average of the crystalline phase band structure. A rough angular average of the $\pi$ (dashed line) and $\sigma$ (solid lines) bands of graphite are shown in the figure. An angular average of the diamond $\sigma$ bands is qualitatively similar to the averaged $\sigma$ bands in graphite except that the bandwidth for diamond is $21 \mathrm{eV}$ while the width of the $\sigma$ bands in graphite is $18 \mathrm{eV}$. It can be seen that the "band structure" of $i$-C is very similar to an angular average of the graphite band structure. The band structure of $e-\mathrm{C}$ is also much like graphite. ${ }^{12} \mathrm{We}$ can estimate the fraction of $s p^{2}$ and $s p^{3}$ bonding in $e$-C and $i$-C from the normalization condition on the spectral momentum density as is discussed next.

The integral of the spectral densities for the $\sigma$ bands in graphite gives three electrons per carbon while the integral over the $\pi$ band gives one electron. The integral of the spectral densities for the four $\sigma$ bands in diamond would give four electrons. If we assume the band in $\alpha$-C at $\sim 8 \mathrm{eV}$ arises from the $\pi$-electron system and assume the dispersing band is associated with the $\sigma$ bands of either graphite or diamond (or some mixture of the two), then according to the model proposed above $e^{1,3}$ in which only pure $s p^{2}$ and $s p^{3}$ bonds exist, the concentration, $c_{s p^{2}}$, of $s p^{2}$ bonds in $\alpha-\mathrm{C}$ can be estimated from the ratio

$$
\frac{N_{\pi, \alpha-\mathrm{C}}(q)}{N_{\sigma, \alpha-\mathrm{C}}(q)}=\frac{c_{s p^{2}} N_{\pi, G}(q)}{c_{s p^{2} N_{\sigma, G}}(q)+\left(1-c_{s p^{2}}\right) N_{\sigma, D}(q)},
$$

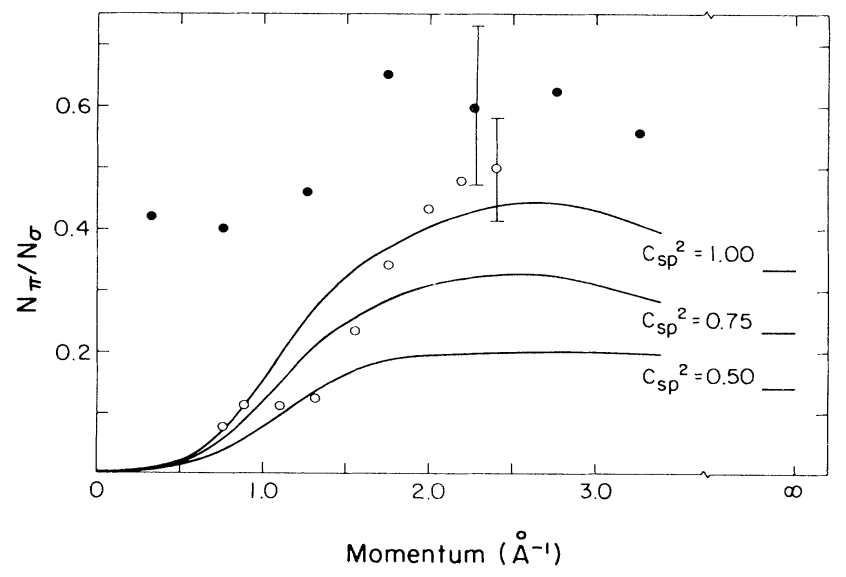

FIG. 4. The ratio of the integrated spectral intensities, $N_{i}(q)=\int d E^{\prime} \iint^{g} 4 \pi q^{\prime 2} d q^{\prime} \rho_{i}\left(E^{\prime}, q^{\prime}\right)$, for the " $\pi$ " and " $\sigma$ " bands of evaporated carbon (open circles) and ion-sputtered carbon (solid circles) as a function of momentum. The solid lines are theoretical predictions of this ratio.

where

$$
N_{\beta, \gamma}(q)=\int_{-\infty}^{\infty} d E^{\prime} \int_{0}^{q} d \Omega d q^{\prime} q^{\prime 2} \rho_{\beta, \gamma}\left(E^{\prime}, \mathbf{q}^{\prime}\right),
$$

$\beta=\pi$ or $\sigma, \gamma=\alpha-\mathrm{C}(e-\mathrm{C}$ or $i-\mathrm{C}), G$ denotes graphite, and $D$ denotes diamond. In the limit $q \rightarrow \infty$, this ratio is $c_{s p^{2}} /\left(4-c_{s p^{2}}\right)$. Unfortunately, we are unable to integrate the spectral density of $i$-C beyond $q=3.5 \AA^{-1}(q \approx 2.5$ $\AA^{-1}$ for $e$-C) because the intensity is lost in the statistical noise. The spectral density of graphite and diamond has been calculated for certain high-symmetry directions by a first-principles, density-functional technique. We estimated $N_{\beta, G}(q)$ and $N_{\sigma, D}(q)$ by smoothly interpolating $\rho_{\beta, \gamma}(E, \mathbf{q})$ between the symmetry directions. The experimental and theoretical results for the ratio of $\pi$-to- $\sigma$ intensity are shown in Fig. 4. The open and solid circles are the experimental results for $e-\mathrm{C}$ and $i$-C, respectively. The solid lines are the theoretical ratios for the three different concentrations of $s p^{2}$ bonding given in the figure. Note the break in the horizontal axis and the exact values of the ratios for $q=\infty$. The experimental ratio is very sensitive to statistical fluctuations at low momentum, but for $q \geq 2.0 \AA^{-1}$ the ratio for both $e-\mathrm{C}$ and $i-\mathrm{C}$ have stabilized to values consistent with $c_{s p^{2}} \approx 1.0$. Given the statistical uncertainty, we estimate that $0.85 \leq c_{s p^{2}} \leq 1.0$ for $e-\mathrm{C}$ and $0.75 \leq c_{s p^{2}} \leq 1.0$ for $i$-C.

Finally, we address the question of why $e-\mathrm{C}$ is soft and black while $i$-C is hard and transparent if the local bonding in both cases is $\sim 100 \% s p^{2}$. Part of the difference, undoubtedly, is due to subtle differences in the electronic structure of the $\pi$ band near the Fermi energy which we cannot resolve. But there is a major difference between the $\pi$-band spectral densities of $e-\mathrm{C}$ and $i$-C which may be an important factor explaining the disparate proper- 
ties of the two materials. We find that the spectral density of the $\pi$ band in $e$-C goes to zero as $q \rightarrow 0$ and has nearly the same momentum dependence as an angular average of the spectral density of the graphite $\pi$ orbital. ${ }^{16}$ In contrast, the momentum density of the $\pi$ orbital in $i$-C (see Fig. 3) is finite at $q=0$ and has the characteristics of a hybridized $s-p$ wave function. In $i-\mathrm{C}$ a distribution of radii for the curvature of the $s p^{2}$ bonded system presumably exists so that the $\pi$-band spectral density is some average over different degrees of rehybridization. A quantitative analysis of this rehybridization will require a more detailed model for the structure of $i-\mathrm{C}$.

In summary, we have argued that the sum rule on the complex dielectric function of $\alpha-\mathrm{C}$ does not give a reliable estimate of the relative proportion of $s p^{2}$ and $s p^{3}$ bonding. We have measured the spectral density of diamondlike $i$-C by $(e, 2 e)$ spectroscopy and find that it has band structure which is qualitatively similar to graphite. From the normalization condition on the spectral density, we estimate that the local bonding in $e-\mathrm{C}$ and $i-\mathrm{C}$ is nearly $100 \% s p^{2}$. In $e$-C the momentum density of the $\pi$ orbital is in agreement with an angular average of the graphite $\pi$-orbital momentum density. The momentum density of the $\pi$ orbital in $i$-C displays significant rehybridization which, we suggest, is due to wrinkling and twisting of the $s p^{2}$ bonded sheets.

We gratefully acknowledge the preparation and characterization of the ion-sputtered samples by T. J. Fabish and J. A. Nemetz-Kelso at Alcoa Technical Center. Theoretical calculations of the spectral density of graphite and diamond were done by N. A. W. Holzwarth at Wake Forest University. This research was supported in part by grants from the Aluminum Company of America and the Virginia Center for Innovative Technology and by Grant No. DMR-8420555 from the National Science Foundation.

${ }^{1}$ J. Robertson, Adv. Phys. 35, 317 (1986), and references therein.

${ }^{2}$ J. C. Angus and C. C. Hayman, Science 241, 913 (1988).

${ }^{3}$ N. Wada, P. J. Gaczi, and S. A. Solin, J. Non-Cryst. Solids 35, 543 (1980).

${ }^{4}$ N. Savvides, J. Appl. Phys. 59, 4133 (1986).

${ }^{5}$ J. Fink et al., Phys. Rev. B 30, 4713 (1984).

${ }^{6}$ E. A. Taft and H. R. Philipp, Phys. Rev. 138, A197 (1965).

${ }^{7}$ R. Sonnenschein, M. Hanfland, and K. Syassen, Phys. Rev. B 38, 3152 (1988). See footnote 30.

${ }^{8}$ R. C. Haddon, L. E. Brus, and Krishnan Raghavachari, Chem. Phys. Lett. 131, 165 (1986).

${ }^{9}$ Giulia Galli, Richard M. Martin, Roberto Car, and Michele Parrinello, Phys. Rev. Lett. 62, 555 (1989).

${ }^{10} \mathrm{H}$. Venghaus, Phys. Status Solidi (b) 71, 609 (1975).

${ }^{11}$ Chao Gao et al., Phys. Rev. B 37, 3914 (1988).

${ }^{12}$ A. L. Ritter, J. R. Dennison, and R. Jones, Phys. Rev. Lett. 53, 2054 (1984).

${ }^{13}$ R. Jones and A. L. Ritter, J. Electron Spectros. Relat. Phenom. 40, 285 (1986).

${ }^{14}$ J. L. Beeby and T. M. Hayes, Phys. Rev. B 32, 6464 (1985).

${ }^{15}$ Roger Haydock, in Current Trends in the Physics of Materials, edited by M. Yussouff (World Scientific, Singapore, 1987), p. 370.

${ }^{16} \mathrm{We}$ have taken more data on evaporated carbon than are presented in Ref. 12. It will be published in a comprehensive article. 